\title{
High bone concentrations of homocysteine are associated with altered bone morphology in humans
}

\author{
Joerg H. Holstein ${ }^{1,2 *}$, Markus Herrmann ${ }^{3,4}$, Christina Splett ${ }^{1,2}$, Wolfgang Herrmann ${ }^{3}$, \\ Patric Garcia ${ }^{1,2}$, Tina Histing ${ }^{1,2}$, Moritz Klein ${ }^{1,2}$, Karsten Kurz ${ }^{5}$, Thomas Siebel ${ }^{5}$, Tim Pohlemann ${ }^{1}$ \\ and Michael D. Menger ${ }^{2}$ \\ ${ }^{1}$ Department of Trauma, Hand and Reconstructive Surgery, University of Saarland, 66421 Homburg/Saar, Germany \\ ${ }^{2}$ Institute for Clinical and Experimental Surgery, University of Saarland, Homburg/Saar, Germany \\ ${ }^{3}$ Department of Clinical Chemistry and Laboratory Medicine, University of Saarland, Homburg/Saar, Germany \\ ${ }^{4}$ Department of Clinical Biochemistry, Royal Prince Alfred Hospital, University of Sydney, Sydney, Australia \\ ${ }^{5}$ Department of Orthopaedic Surgery, Knappschaftskrankenhaus Püttlingen, Püttlingen, Germany
}

(Received 29 August 2010 - Revised 17 January 2011 - Accepted 17 January 2011 - First published online 27 April 2011)

\begin{abstract}
Accumulation of homocysteine and S-adenosylhomocysteine in bone has been shown to be associated with reduced bone quality in rats. The aim of the present study was to investigate whether high bone concentrations of homocysteine and $S$-adenosylhomocysteine as well as a low methylation capacity are related to an impaired bone morphology in humans. Concentrations of homocysteine and its precursors $S$-adenosylhomocysteine and $S$-adenosylmethionine were measured in femoral bone samples of eighty-two males and females (age 71 (SD 8) years) who underwent elective hip arthroplasty. Cancellous bone structure was analysed by histomorphometry. In addition, blood was sampled to measure serum concentrations of homocysteine. Results of bone and serum analyses were grouped for individuals with high or low bone concentrations of homocysteine, $S$-adenosylhomocysteine and $S$-adenosylmethionine, as well as for individuals with a high or a low methylation capacity, which is indicated by a low or a high $S$-adenosylhomocysteine:S-adenosylmethionine ratio $(n 41$, each). Histomorphometry showed a higher trabecular separation and a lower trabecular thickness, trabecular number and trabecular area in individuals with high bone concentrations of homocysteine and $S$-adenosylhomocysteine compared with individuals with low bone concentrations of homocysteine and $S$-adenosylhomocysteine. There was no association between the $S$-adenosylhomocysteine: $S$-adenosylmethionine ratio and bone morphology. It was found that $48 \%$ of bone homocysteine was bound to the collagen of the extracellular bone matrix. Blood analyses demonstrated a significant correlation between serum and bone homocysteine. The results of the present study indicate an association between altered bone morphology and elevated bone concentrations of homocysteine and $S$-adenosylhomocysteine, but not between altered bone morphology and methylation capacity.
\end{abstract}

Key words: Homocysteine: $S$-adenosylhomocysteine: $S$-adenosylmethionine: Histomorphometry: Bone morphology

The aim of the present study was to investigate whether high tissue concentrations of homocysteine (HCY) and its precursors $S$-adenosylhomocysteine (SAH) and $S$-adenosylmethionine (SAM) in human bone are associated with altered bone morphology. Additionally, we aimed to assess the association between cancellous bone structure and methylation capacity, which is indicated by the SAH:SAM ratio.

In rats, high bone concentrations of HCY and SAH as well as an increased SAH:SAM ratio have been shown to be associated with a reduced bone quality ${ }^{(1)}$. A recently published in vitro study has further demonstrated that decreased SAMdependent methylation impairs osteoblast differentiation ${ }^{(2)}$.
Therefore, our hypothesis was that high bone concentrations of HCY and SAH, an increased SAH:SAM ratio as well as a low bone concentration of SAM are associated with a deteriorated cancellous bone structure also in humans.

\section{Methods}

Study design

We harvested cancellous bone samples at the femoral neck of eighty-two osteoarthritis patients undergoing hip arthroplasty. Cancellous bone structure was analysed by

Abbreviations: HCY, homocysteine; SAH, S-adenosylhomocysteine; SAM, S-adenosylmethionine; Tb.Ar, trabecular area; Tb.N, trabecular number; Tb.Sp, trabecular separation; Tb.Th, trabecular thickness.

*Corresponding author: J. H. Holstein, fax +496841 16 31503, email joerg.holstein@uks.eu 
histomorphometry. In addition, we measured bone concentrations of total HCY, collagen-bound HCY, SAH and SAM. Blood was sampled to assess the serum concentration of HCY. For each bone metabolite, we calculated the median. In addition, we determined the median bone SAH:SAM ratio. These values were used as cut-off points. The subjects with values above the median were assigned to the high category groups, and the subjects with values less than the median were assigned to the low category groups.

\section{Subjects}

Subjects were recruited from the Orthopaedic Department of the Knappschaftskrankenhaus Püttlingen, Germany, during the time period from 1 March 2006 until 31 January 2007. The age range of the study population was 44-86 years. None of the patients had hip injury or had been immobilised. Individuals with non-normal serum concentrations of $\mathrm{Ca}$ and vitamin D were not included in the study. Subjects were also excluded from the study if they suffered from hyper- or hypoparathyroidism, hyper- or hypothyroidism, malignancies and inflammatory diseases, or if they received one of the following medications: oestrogen, parathormone, vitamin $\mathrm{D}$, strontium ranelate, bisphosphonates, corticosteroids, anticonvulsants and cytostatic or immunosuppressive drugs. The present study was conducted according to the guidelines laid down in the Declaration of Helsinki, and all procedures involving human subjects were approved by the Institutional Review Board and Ethical Commission of the University of Saarland (Homburg/Saar, Germany). Written informed consent was obtained from all subjects.

\section{Specimen preparation and blood sampling}

Bone samples were fixed in $4 \%$ phosphate-buffered formalin for histomorphometric evaluation or stored at $-80^{\circ} \mathrm{C}$ for the analyses of HCY, SAH and SAM concentrations.

Fasting blood was collected in serum tubes $1 \mathrm{~d}$ before surgery between 08.00 and 10.00 hours. After $20 \mathrm{~min}$ of clotting, samples were centrifuged at $4000 \mathrm{rpm}$ for $10 \mathrm{~min}$ to separate serum from cellular blood components. Blood serum was stored at $-80^{\circ} \mathrm{C}$ until measurement of HCY.

\section{Histomorphometry}

Formalin-fixed bone samples were decalcified in 10\% EDTA solution for 5 weeks and embedded in paraffin. Using haematoxylin-eosin-stained sections of $5 \mu \mathrm{m}$ thickness, cancellous bone structure was assessed at a magnification of $4 \times$ (Olympus BX60 Microscope; Olympus, Tokyo, Japan; Zeiss Axio Cam and Axio Vision 3.1; Carl Zeiss, Oberkochen, Germany; ImageJ; NIH, Bethesda, MD, USA). The following histomorphometric parameters were determined and calculated in adherence to the recommendations of the American Society of Bone and Mineral Research: trabecular thickness (Tb.Th); trabecular number (Tb.N); trabecular separation (Tb.Sp); percentage of trabecular area (Tb.Ar) ${ }^{(3)}$. The quantitative analysis of Tb.Th, Tb.N, Tb.Sp and Tb.Ar provides important information on the quality of cancellous bone. Accordingly, a decrease in Tb.Th, Tb.N and Tb.Ar and an increase in Tb.Sp can indicate a reduced bone quality.

\section{Bone analyses of homocysteine, S-adenosylhomocysteine and S-adenosy/methionine}

Collagen-bound HCY was extracted from the bone samples using a collagenase digestion method as reported previously $^{(1)}$. Concentrations of total HCY and collagen-bound HCY in bone were measured by HPLC (Agilent 1100; Waldbronn, Germany) using a fluorescence detector and a commercial assay (Immundiagnostik, Bensheim, Germany) ${ }^{(1)}$

SAM and SAH were measured in the bone samples using a modified liquid chromatography-tandem MS method as described previously ${ }^{(1,4)}$. Inter-assay CV were $3.8 \%$ for HCY, $8.0 \%$ for SAH and $4.8 \%$ for SAM.

\section{Serum analysis of homocysteine}

HCY was analysed with an enzymatic fluorescence polarisation immunoassay on an AXSYM automated analyser (Abbott, Wiesbaden, Germany). Intra- and inter-assay CV were 4.5 and $4.6 \%$, respectively.

\section{Statistics}

All data are given as means and standard deviations. The comparison between the experimental groups was performed by Student's $t$ test for those outcome variables that were normally distributed and by the Mann-Whitney $U$ test for those outcome variables that were not normally distributed. Comparison of different categories between the groups was performed by the $\chi^{2}$ test or Fisher's exact test. Spearman's rank correlation coefficient was used to determine the strength of association between serum and bone concentrations of HCY. A $P$ value $<0.05$ was considered to indicate significant differences. All statistical analyses were performed using the SPSS software package, version 17.0 for Windows (SPSS, Inc., Chicago, IL, USA).

\section{Results}

Bone analyses of homocysteine, S-adenosylhomocysteine and S-adenosylmethionine

Median bone concentrations of HCY, SAH and SAM were $0.0546,0.0159$ and $0.0836 \mu \mathrm{mol} / 1$, respectively. The interquartile ranges of bone metabolite concentrations were 0.0405$0.0732 \mu \mathrm{mol} / 1$ for HCY, $0.0113-0.0243 \mu \mathrm{mol} / 1$ for SAH and $0 \cdot 0450-0 \cdot 1326 \mu \mathrm{mol} / 1$ for SAM. Based on the medians, individuals were grouped into high concentrations of $\mathrm{HCY}$ $\left(\mathrm{HCY}^{+}\right.$group, 0.0547-0.0937 $\left.\mu \mathrm{mol} / \mathrm{l}\right), \mathrm{SAH}\left(\mathrm{SAH}^{+}\right.$group, $0.0161-0.1712 \mu \mathrm{mol} / \mathrm{l})$ and $\mathrm{SAM}\left(\mathrm{SAM}^{+}\right.$group, 0.0837$0.5743 \mu \mathrm{mol} / \mathrm{l}$ ) or low concentrations of HCY ( $\mathrm{HCY}^{-}$group, $0.0031-0.0545 \mu \mathrm{mol} / \mathrm{l}), \quad \mathrm{SAH} \quad\left(\mathrm{SAH}^{-}\right.$group, $0.0054-$ $0.0157 \mu \mathrm{mol} / \mathrm{l})$ and SAM (SAM ${ }^{-}$group, 0.0039-0.0835 $\left.\mu \mathrm{mol} / \mathrm{l}\right)$. The median SAH:SAM ratio was 0.206 with a range of 
$0.030-0.205$ in the high-methylation capacity group (SAH:SAM ${ }^{-}$group) and $0.207-3.262$ in the low-methylation capacity group (SAH:SAM ${ }^{+}$group). It was found that $48 \%$ of the total HCY was bound to collagen.

\section{Anthropometric characteristics}

Individuals of the $\mathrm{SAH}^{+}$group showed a significantly lower BMI compared with individuals of the $\mathrm{SAH}^{-}$group. In the $\mathrm{SAM}^{+}$group, the number of females was significantly higher than that in the $\mathrm{SAM}^{-}$group. No significant differences in the anthropometric characteristics were found between individuals of the $\mathrm{HCY}^{+}$and $\mathrm{HCY}^{-}$groups as well as between the individuals of the SAH:SAM ${ }^{+}$and $\mathrm{SAH}_{\mathrm{SAM}}{ }^{-}$groups (Table 1).

\section{Serum analysis of homocysteine}

Mean serum concentrations of HCY were 11.7 (SD 3.1) $\mu \mathrm{mol} / 1$ in the low and $14.1(\mathrm{SD} 6.7) \mu \mathrm{mol} / \mathrm{l}$ in the high-HCY bone concentration groups, 11.9 (SD 3.1) $\mu \mathrm{mol} / \mathrm{l}$ in the low- and 13.8 (SD 6.7$) \mu \mathrm{mol} / 1$ in the high-SAH bone concentration groups, as well as 13.2 (SD 6.8) $\mu \mathrm{mol} / \mathrm{l}$ in the low- and 12.5 (SD $3 \cdot 2) \mu \mathrm{mol} / 1$ in the high-SAM bone concentration groups. Correlation analysis showed a significant correlation between the serum and bone concentrations of HCY $(r 0 \cdot 26, P=0.03)$.

\section{Histomorphometry}

Individuals with high HCY bone concentrations demonstrated a significantly higher Tb.Sp compared with individuals with low-HCY bone concentrations. In the $\mathrm{SAH}^{+}$group, Tb.N and $\mathrm{Tb} . \mathrm{Ar}$ were significantly lower than in the $\mathrm{SAH}^{-}$group. In $\mathrm{SAM}^{+}$individuals, Tb.Th, Tb.N and Tb.Ar were significantly lower, and Tb.Sp was significantly higher compared with individuals of the $\mathrm{SAM}^{-}$group. In contrast, there were no significant differences in bone morphology between individuals of the SAH:SAM ${ }^{+}$and SAH:SAM ${ }^{-}$groups (Table 1).

\section{Discussion}

To the best of our knowledge, the present study reports for the first time on tissue concentrations of HCY and its precursors in human bone. Our first hypothesis was that high tissue concentrations of HCY and SAH are related to a deteriorated cancellous bone structure in humans. The results of the present study confirm this hypothesis, showing a positive correlation between high tissue concentrations of HCY and SAH in bone and a deteriorated cancellous bone structure. Our second hypothesis was that low tissue concentrations of SAM as well as a high SAH:SAM ratio in bone are associated with an impaired cancellous bone structure in humans. In contrast to our first hypothesis, we had to reject this second hypothesis, because we did not find a significant association between the SAH:SAM ratio and cancellous bone structure, and because a low SAM concentration was not associated with an impaired but an improved cancellous bone structure. This result is surprising and seemingly contradictory to the results of previous in vitro and animal studies but may be explained by the role of B vitamins in HCY metabolism. The $\mathrm{B}$ vitamins folate, $\mathrm{B}_{6}$ and $\mathrm{B}_{12}$ are important cofactors of the HCY-degrading remethylation and trans-sulfuration pathways $^{(5)}$. Accordingly, deficiency of these $\mathrm{B}$ vitamins is the main cause of increased serum and probably also bone

Table 1. Anthropometric characteristics and histomorphometric data of the total study population and of individuals with high or low bone concentrations of homocysteine (HCY), S-adenosylhomocysteine (SAH) and S-adenosylmethionine (SAM) as well as a low or high methylation capacity ${ }^{*}$

(Mean values, standard deviations and percentages)

\begin{tabular}{|c|c|c|c|c|c|c|c|c|c|c|c|c|c|c|}
\hline & \multicolumn{6}{|c|}{ Anthropometric characteristics } & \multicolumn{8}{|c|}{ Histomorphometry $†$} \\
\hline & \multirow[b]{2}{*}{ Female (\%) } & \multicolumn{2}{|c|}{ Age (years) } & \multicolumn{2}{|c|}{ BMl $\left(\mathrm{kg} / \mathrm{m}^{2}\right)$} & \multirow[b]{2}{*}{ Smokers (\%) } & \multicolumn{2}{|c|}{ Tb.Th (mm) } & \multicolumn{2}{|c|}{ Tb.N (per mm) } & \multicolumn{2}{|c|}{ Tb.Sp (mm) } & \multicolumn{2}{|c|}{ Tb.Ar (\%) } \\
\hline & & Mean & SD & Mean & SD & & Mean & SD & Mean & SD & Mean & SD & Mean & SD \\
\hline Totalł & 63 & 71 & 8 & 27.5 & 4.3 & 8 & 0.32 & 0.12 & 0.33 & 0.13 & $2 \cdot 5$ & 0.7 & $12 \cdot 0$ & $6 \cdot 4$ \\
\hline $\mathrm{HCY}^{+}$ & 73 & 70 & 9 & $27 \cdot 1$ & 4.4 & 8 & 0.31 & $0 \cdot 12$ & 0.31 & 0.14 & $2 \cdot 7$ & 0.8 & $11 \cdot 3$ & $6 \cdot 7$ \\
\hline $\mathrm{HCY}^{-}$ & 54 & 71 & 6 & 27.9 & 4.3 & 8 & 0.33 & $0 \cdot 12$ & 0.36 & 0.11 & $2 \cdot 4$ & 0.6 & $12 \cdot 7$ & $6 \cdot 0$ \\
\hline$P \S$ & 0.11 & \multicolumn{2}{|c|}{0.85} & \multicolumn{2}{|c|}{0.67} & 1.00 & \multicolumn{2}{|c|}{0.41} & \multicolumn{2}{|c|}{0.15} & \multicolumn{2}{|c|}{0.04} & \multicolumn{2}{|c|}{0.19} \\
\hline $\mathrm{SAH}^{+}$ & 68 & 70 & 8 & $26 \cdot 2$ & $3 \cdot 6$ & 8 & 0.31 & 0.13 & 0.31 & 0.13 & $2 \cdot 6$ & 0.7 & $10 \cdot 7$ & $6 \cdot 4$ \\
\hline $\mathrm{SAH}^{-}$ & 59 & 70 & 10 & $28 \cdot 8$ & 4.6 & 8 & 0.34 & 0.13 & 0.37 & 0.14 & $2 \cdot 4$ & 0.8 & $14 \cdot 1$ & 7.6 \\
\hline$P \S$ & 0.49 & \multicolumn{2}{|c|}{0.77} & \multicolumn{2}{|c|}{0.01} & 1.00 & \multicolumn{2}{|c|}{0.12} & \multicolumn{2}{|c|}{0.03} & \multicolumn{2}{|c|}{0.15} & \multicolumn{2}{|c|}{0.01} \\
\hline SAM $^{+}$ & 80 & 72 & 8 & $27 \cdot 2$ & 4.6 & 11 & 0.29 & $0 \cdot 12$ & 0.30 & 0.14 & $2 \cdot 8$ & 0.8 & $10 \cdot 2$ & $6 \cdot 0$ \\
\hline $\mathrm{SAM}^{-}$ & 46 & 69 & 10 & $27 \cdot 9$ & $4 \cdot 1$ & 5 & 0.35 & $0 \cdot 13$ & 0.36 & 0.12 & $2 \cdot 3$ & 0.6 & $14 \cdot 1$ & 7.5 \\
\hline & $<0.01$ & \multicolumn{2}{|c|}{0.14} & \multicolumn{2}{|c|}{0.48} & 0.67 & \multicolumn{2}{|c|}{0.03} & \multicolumn{2}{|c|}{0.04} & \multicolumn{2}{|c|}{0.01} & \multicolumn{2}{|c|}{$<0.01$} \\
\hline SAH:SAM ${ }^{+}$ & 73 & 70 & 8 & $27 \cdot 1$ & $4 \cdot 2$ & 8 & 0.32 & $0 \cdot 13$ & 0.32 & 0.11 & $2 \cdot 5$ & 0.6 & $12 \cdot 0$ & $6 \cdot 4$ \\
\hline SAH:SAM ${ }^{-}$ & 54 & 72 & 9 & $28 \cdot 0$ & 4.5 & 8 & 0.31 & $0 \cdot 12$ & 0.34 & 0.15 & $2 \cdot 6$ & 0.9 & 11.9 & 6.5 \\
\hline$P \S$ & 0.11 & \multicolumn{2}{|c|}{0.24} & \multicolumn{2}{|c|}{0.35} & 1.00 & \multicolumn{2}{|c|}{0.91} & \multicolumn{2}{|c|}{0.51} & & & \multicolumn{2}{|c|}{0.91} \\
\hline
\end{tabular}

Tb.Th, trabecular thickness; Tb.N, trabecular number; Tb.Sp, trabecular separation; Tb.Ar, trabecular area.

* Comparisons between the experimental groups were performed by the $\chi^{2}$ test (male and female), the Mann-Whitney $U$ test (age, Tb.Th and Tb.Ar), Student's $t$ test (BMI, Tb.N and Tb.Sp) or Fisher's exact test (smokers).

$\dagger$ Histomorphometric parameters include Tb.Th, Tb.N, Tb.Sp and Tb.Ar.

¥ The medians of the total study population (total) were defined as cut-off values indicating high (+) or low (-) bone concentrations of HCY, SAH and SAM as well as a low or high methylation capacity (SAH:SAM ${ }^{+} v$. SAH:SAM ${ }^{-}$). Total, $n 82 . \mathrm{HCY}^{+}, \mathrm{HCY}^{-}, \mathrm{SAH}^{+}, \mathrm{SAH}^{-}, \mathrm{SAM}^{+}, \mathrm{SAM}^{-}, \mathrm{SAH}_{\mathrm{SAM}}^{+}$and SAH:SAM ${ }^{-} \mathrm{groups} n 41$ (each). 
concentrations of $\mathrm{HCY}^{(5,6)}$. Furthermore, a reduced metabolism of HCY caused by B vitamin deficiency leads to an accumulation of its precursors $\mathrm{SAH}$ and $\mathrm{SAM}^{(5)}$. Therefore, we suggest that high bone SAM concentrations are a consequence of high concentrations of SAH and HCY. This relationship might explain why not only high concentrations of HCY and SAH but also of SAM are related to a deteriorated cancellous bone structure.

We could demonstrate that about $50 \%$ of $\mathrm{HCY}$ is bound to collagen in human bone. It has been shown in vitro that HCY is capable of binding to aldehyde groups of collagen, and thereby affecting the formation of stable collagen crosslinks ${ }^{(6)}$. Of interest, collagen $\mathrm{I}$ is the dominant protein of the organic bone matrix. In accordance, a disturbance of collagen cross-linking through HCY might be one explanation for the alteration of bone morphology. Apart from a disturbed collagen cross-linking, a HCY-induced stimulation of osteoclasts has also been discussed as one possible pathomechanism explaining the adverse effects of HCY on bone ${ }^{(6)}$.

According to a review by Herrmann et al. ${ }^{(6)}$ on the role of hyperhomocysteinaemia in osteoporosis, the majority of large epidemiological trials found a significant positive relationship between serum HCY concentrations and fracture risk. In contrast, the association between hyperhomocysteinaemia and decreased bone mineral density is controversially discussed. About half of all studies, including a total of almost 20000 individuals, found a significant inverse relationship between serum HCY and bone mineral density ${ }^{(6)}$. Existing data of analyses of bone turnover markers suggest a shift in bone metabolism towards bone resorption ${ }^{(7)}$. So far, two large intervention studies have investigated whether a HCYlowering treatment with B vitamins is capable of decreasing the incidence of osteoporotic fractures ${ }^{(6,8)}$. In the first study, which included 615 individuals, fracture risk was found to be significantly reduced in patients receiving $\mathrm{B}$ vitamin supplementation, while the second study, which included more than 5000 patients, could not demonstrate a fracturepreventing effect of B vitamin supplementation. In summary, the entirety of data derived from epidemiological and interventional studies analysing the association between hyperhomocysteinaemia and fracture risk, bone mineral density as well as serological bone turnover markers, is conflicting. The data of the present study indicate a negative bone turnover in patients with high tissue concentrations of HCY in bone, while the deteriorated cancellous bone structure might be additionally an indication of an increased fracture risk. Thus, the results of the present study confirm the data of those epidemiological studies, which have reported an association between hyperhomocysteinaemia and a negative bone turnover as well as an increased risk of osteoporotic fractures.

Anthropometric data of the present study indicate a significantly lower BMI of individuals with high bone concentrations of SAH compared with individuals with low bone concentrations of SAH. These results indicate a relationship but do not prove causality between bone SAH and BMI. In mice, it has been shown that the application of a methyl-deficient diet prevents weight gain and increases $\mathrm{SAH}$ concentrations in a variety of different tissues ${ }^{(9)}$. However, these data do not allow conclusions on whether changes in body weight were dependent on tissue concentrations of $\mathrm{SAH}$ or other effects of methyl deficiency. In humans, it has been shown that osteoporosis is related - beside other factors - also to decreased body weight ${ }^{(10)}$. This relation is reflected by the results of the present study demonstrating that high bone concentrations of SAH are associated with both lower BMI and deteriorated cancellous bone structure. Furthermore, it has to be assumed that total bone mass is decreased in osteoporotic patients, which in turn might also affect total body weight and thus $\mathrm{BMI}^{(10)}$. Accordingly, it might be possible that the decrease in cancellous bone mass in individuals with high bone concentrations of SAH results in a reduced BMI.

A significant difference in sex distribution has been found between the $\mathrm{SAM}^{+}$and $\mathrm{SAM}^{-}$groups, showing a significantly higher number of women in the $\mathrm{SAM}^{+}$group than in the $\mathrm{SAM}^{-}$group. For rats, it has been reported that the activity of the methionine-activating enzyme methionine adenosyltransferase is higher in the liver of female than in that of male animals ${ }^{(11)}$. To the best of our knowledge, there is no information on a sex-dependent activity of methionine adenosyltransferase in humans.

Some limitations of the present study have to be considered. As subjects who were recruited for the present study underwent hip arthroplasty due to osteoarthritis, it has to be addressed that bone of the proximal femur might differ between hip osteoarthritis patients and non-hip osteoarthritis patients $^{(12)}$. A second limitation is that the mean age of the study population was relatively high (71 (SD 8) years), which might explain the relatively high mean serum concentration of $\mathrm{HCY}^{(13)}$.

We conclude that high tissue concentrations of HCY and SAH in bone are related to reduced bone quality, while the relationship between SAM and altered bone properties is most probably only affected in situations of high HCY and SAH concentrations. We further conclude that altered methylation capacity is not associated with deteriorated bone morphology.

\section{Acknowledgements}

We thank Janine Becker for excellent technical assistance. All authors have no conflicts of interest. The present study was financed by the Institute for Clinical and Experimental Surgery, University of Saarland. The present study received no specific grant from any funding agency in the public, commercial or not-for-profit sectors. The authors' contributions were as follows: J. H. H., M. H., W. H., T. S., T. P. and M. D. M. designed the study; J. H. H., M. H., C. S., P. G., T. H., M. K., K. K., T. S., T. P. and M. D. M. conducted the study; W. H., T. S., T. P. and M. D. M. provided essential reagents or provided essential materials; J. H. H., M. H., C. S., W. H. and M. D. M. analysed the data or performed the statistical analysis; J. H. H., M. H. and M. D. M. wrote the manuscript; J. H. H., M. H., T. P. and M. D. M. had the primary responsibility for the final content. All authors read and approved the final manuscript. 


\section{References}

1. Herrmann M, Tami A, Wildemann B, et al. (2009) Hyperhomocysteinemia induces a tissue specific accumulation of homocysteine in bone by collagen binding and adversely affects bone. Bone 44, 467-475.

2. Vaes BL, Lute C, van der Woning SP, et al. (2010) Inhibition of methylation decreases osteoblast differentiation via a nonDNA-dependent methylation mechanism. Bone $\mathbf{4 6}$, 514-523.

3. Parfitt AM, Drezner MK, Glorieux FH, et al. (1987) Bone histomorphometry: standardization of nomenclature, symbols, and units. Report of the ASBMR Histomorphometry Nomenclature Committee. J Bone Miner Res 2, 595-610.

4. Gellekink H, van Oppenraaij-Emmerzaal D, van Rooij RA, et al. (2005) Stable-isotope dilution liquid chromatography-electrospray injection tandem mass spectrometry method for fast, selective measurement of $S$-adenosylmethionine and $S$-adenosylhomocysteine in plasma. Clin Chem $\mathbf{5 1}$, $1487-1492$.

5. Herrmann W, Herrmann M \& Obeid R (2007) Hyperhomocysteinaemia: a critical review of old and new aspects. Curr Drug Metab 8, 17-31.

6. Herrmann M, Peter Schmidt J, Umanskaya N, et al. (2007) The role of hyperhomocysteinemia as well as folate, vitamin
$\mathrm{B}(6)$ and $\mathrm{B}(12)$ deficiencies in osteoporosis: a systematic review. Clin Chem Lab Med 45, 1621-1632.

7. Dhonukshe-Rutten RA, Pluijm SM, de Groot LC, et al. (2005) Homocysteine and vitamin $\mathrm{B}_{12}$ status relate to bone turnover markers, broadband ultrasound attenuation, and fractures in healthy elderly people. J Bone Miner Res 20, 921-929.

8. Sawka AM, Ray JG, Yi Q, et al. (2007) Randomized clinical trial of homocysteine level lowering therapy and fractures. Arch Intern Med 167, 2136-2139.

9. Caudill MA, Wang JC, Melnyk S, et al. (2001) Intracellular $S$-adenosylhomocysteine concentrations predict global DNA hypomethylation in tissues of methyl-deficient cystathionine beta-synthase heterozygous mice. J Nutr 131, 2811-2818.

10. Lane JM, Russell L \& Khan SN (2000) Osteoporosis. Clin Orthop Relat Res 372, 139-150.

11. Natori Y (1963) Studies on ethionine. VI. Sex-dependent behavior of methionine and ethionine in rats. $J$ Biol Chem 238, 2075-2080.

12. Dequeker J, Aerssens J \& Luyten FP (2003) Osteoarthritis and osteoporosis: clinical and research evidence of inverse relationship. Aging Clin Exp Res 15, 426-439.

13. Herrmann M, Kraenzlin M, Pape G, et al. (2005) Relation between homocysteine and biochemical bone turnover markers and bone mineral density in peri- and post-menopausal women. Clin Chem Lab Med 43, 1118-1123. 